\title{
El desempeño docente como condición para evitar el plagio académico
}

\author{
Muñoz-Cantero, Jesús-Miguel'; Espiñeira-Bellón, Eva-María ${ }^{2}$ \\ 1Universidade da Coruña, Facultad de Ciencias de la Educación, Código ORCID: 0000-0001- \\ 5502-1771 \\ 2 Universidade da Coruña, Facultad de Ciencias de la Educación, Código ORCID: 0000-0001- \\ 7522-9406
}

\section{RESUMEN $^{\mathrm{i}}$}

El proceso de enseñanza-aprendizaje universitario se produce cuando el profesorado enseña a aprender al estudiantado, de tal manera que el resultado de dichas actividades dé lugar a documentos en los que no se vulnere la honestidad/integridad académica. No obstante, el plagio académico es una de las prácticas deshonestas más extendidas. En esta comunicación se describen y analizan cualitativa y cuantitativamente las causas a las que el alumnado atribuye el plagio académico en función del comportamiento docente. Se cuenta con la participación de 1699 estudiantes de grado y 286 de alumnos de máster de la rama de Ciencias Sociales y Jurídicas de la Universidade da Coruña a quienes se le ha administrado el Cuestionario de Atribuciones para la Detección de Coincidencias en Trabajos Académicos (CUDECO). Los resultados muestran que la baja coordinación entre docentes para evitar la saturación de trabajos y el inadecuado seguimiento, son los factores más relevantes que el alumnado señala como condiciones desfavorables por parte de los/las docentes, asociadas a la comisión de plagio. Como conclusión, con la información obtenida se facilitan estrategias de prevención para mejorar el proceso de enseñanza-aprendizaje universitario, la propia organización y el desempeño docente.

PALABRAS CLAVE: plagio, ética, educación superior, profesorado universitario, ciencias sociales y jurídicas. 


\section{CITA RECOMENDADA:}

Muñoz-Cantero, Jesús-Miguel; Espiñeira-Bellón, Eva-María (2020): El desempeño docente como condición para evitar el plagio académico. En De la Torre Fernández, E. (ed.) (2020). Contextos universitarios transformadores: Boas prácticas no marco dos GID. IV Xornadas de Innovación Docente. Cufie. Universidade da Coruña. A Coruña (págs. 421-434).

DOI capítulo: https://doi.org/10.17979/spudc.9788497497756.421

DOI libro: https://doi.org/10.17979/spudc.9788497497756

\section{ABSTRACT}

The university teaching-learning process occurs when teachers teach students to learn, so that the result of these activities results in documents in which academic honesty / integrity is not violated. However, academic plagiarism is one of the most widespread dishonest practices. In this communication, the causes to which students attribute academic plagiarism based on teaching behavior are described and analyzed qualitatively and quantitatively. It has the participation of 1699 undergraduate students and 286 master students of the branch of Social and Legal Sciences of the University of Coruña who have been administered the Questionnaire of Attributions for the Detection of Coincidences in Academic Works (CUDECO). The results show that the low coordination between teachers to avoid job saturation and inadequate follow-up are the most relevant factors that students indicate as unfavorable conditions for teachers, associated with the plagiarism commission. In conclusion, with the information obtained, prevention strategies are provided to improve the university teaching-learning process, the organization itself and the teaching performance.

KEY WORDS: plagiarism, ethic, higher education, university teaching staff, social and legal sciencies. 


\section{INTRODUCCIÓN}

Las titulaciones universitarias y los estudios superiores se distribuyen en cinco ramas de estudio o conocimiento (Artes y Humanidades, Ciencias, Ciencias de la Salud, Ciencias Sociales y Jurídicas e Ingeniería y Arquitectura). La rama de Ciencias Sociales y Jurídicas agrupa aquellas carreras vinculadas a las Ciencias Sociales como Derecho, Sociología, Economía, Pedagogía, Psicología, ..., aquellas relacionadas con el estudio de la sociedad y de los comportamientos humanos. En dicha rama, el actual proceso de enseñanza-aprendizaje universitario se produce mediante la captación y elaboración de contenidos, ideas, argumentos, información, datos, ... tanto expuestos por el profesorado como a través de cualquier otra fuente de información (Rodríguez, 2007; Universidad Rovira i Virgili, 2017). Además, las metodologías docentes empleadas llevan al alumnado a tener que realizar un gran número de trabajos de carácter autónomo cuyo resultado, en numerosas ocasiones, da lugar a documentos en los que se vulnera la honestidad/integridad académica a través del plagio académico (Sutherland-Smith, 2010; Williams y Carroll, 2009), efectuado por el alumnado cuando "copia el trabajo de otras personas y lo presenta como propio" (Universidad Rovira i Virgili, 2017, p. 4); siendo, por tanto, una forma de deshonestidad o fraude académico, que, en palabras de Diez-Martínez (2015), se produce ante "cualquier comportamiento intelectual que busque un beneficio académico, económico, afectivo o de cualquier otra índole que vaya en contra de los principios éticos de las instituciones educativas" (p. 3).

Se han analizado diversos estudios en los que se tratan de conocer los motivos que llevan al estudiantado universitario de dicha rama a cometer plagio asociados a las características y comportamientos del profesorado universitario durante el proceso de enseñanza-aprendizaje (Amador-Bautista, 2017; Bautista, Sánchez, \& Canto, 2017; Cebrián-Robles, Raposo-Rivas, Cebrián-de-la-Serna, \& Sarmiento Campos, 2018; Comas-Forgas, \& Sureda-Negre, 2016; Diez-Martínez, 2015; Escalante, \& Ibarra, 2013; Sanvicén, \& Molina, 2015; Sureda, Comas, \& Morey, 2009). Entre ellos, pueden citarse los siguientes: la poca claridad en las instrucciones y normas facilitadas, la falta de exigencia del profesorado en la realización de las referencias bibliográficas de forma correcta, la actitud de desinterés o despreocupación del profesorado 
por evitar el plagio, la evaluación del resultado del trabajo sin tener en cuenta el proceso, el tipo de actividades/trabajos solicitados, la falta de antelación a la hora de solicitar los trabajos, la escasa supervisión de los trabajos demandados, la descoordinación entre el profesorado, el número de trabajos solicitados, no motivar al alumnado, no evaluar la correcta citación de fuentes, no manejar herramientas de detección de fragmentos copiados, el peso del trabajo en la nota final, ...

En esta comunicación, se analiza la percepción que tiene el alumnado universitario de la rama de Ciencias Sociales y Jurídicas sobre las actuaciones docentes en cuestiones relativas a la evitación del plagio, ya que, como señalan Hu y Sun (2016), "como expertos académicos y educadores de futuros académicos, los profesores universitarios desempeñan un papel clave en la formación de los estudiantes contra el plagio y en la defensa de la integridad académica" (p. 29).

\section{DESCRICIÓN DE LA EXPERIENCIA}

Para alcanzar el objetivo propuesto, se ha combinado la metodología cuantitativa con la cualitativa. La cuantitativa se desarrolló a través de la técnica de encuestación mediante cuestionario, aplicado al alumnado con el objetivo de que proporcionase la información deseada sobre sus opiniones (García, \& García, 2012) y también teniendo en cuenta que es una técnica de recogida de datos habitual en la investigación educativa, ya que en muchos sondeos mediante encuesta se emplean cuestionarios (McMillan, \& Schumacher, 2005). Dicho cuestionario, contaba con un ítem de carácter abierto que ha permitido desarrollar la metodología de carácter cualitativo.

\section{POBLACIÓN Y MUESTRA}

En el estudio, ha participado el alumnado matriculado en el curso 2018/2019 en los títulos de grado y máster de la rama de Ciencias Sociales y Jurídicas de la UDC. Mediante un muestreo no probabilístico intencional, se accedió a un total de 1699 alumnos/as de trece títulos de grado (figura 1) y 286 alumnos/as de diez títulos de máster (figura 2). 


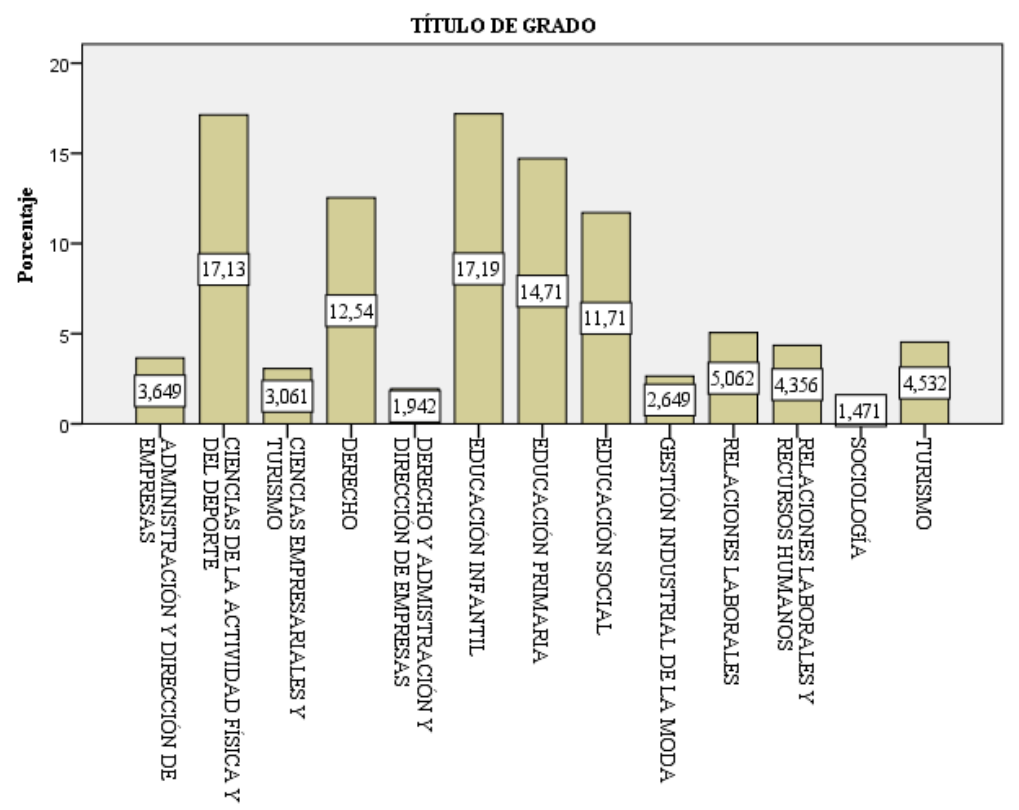

Figura 1. Títulos que cursa el alumnado de grado

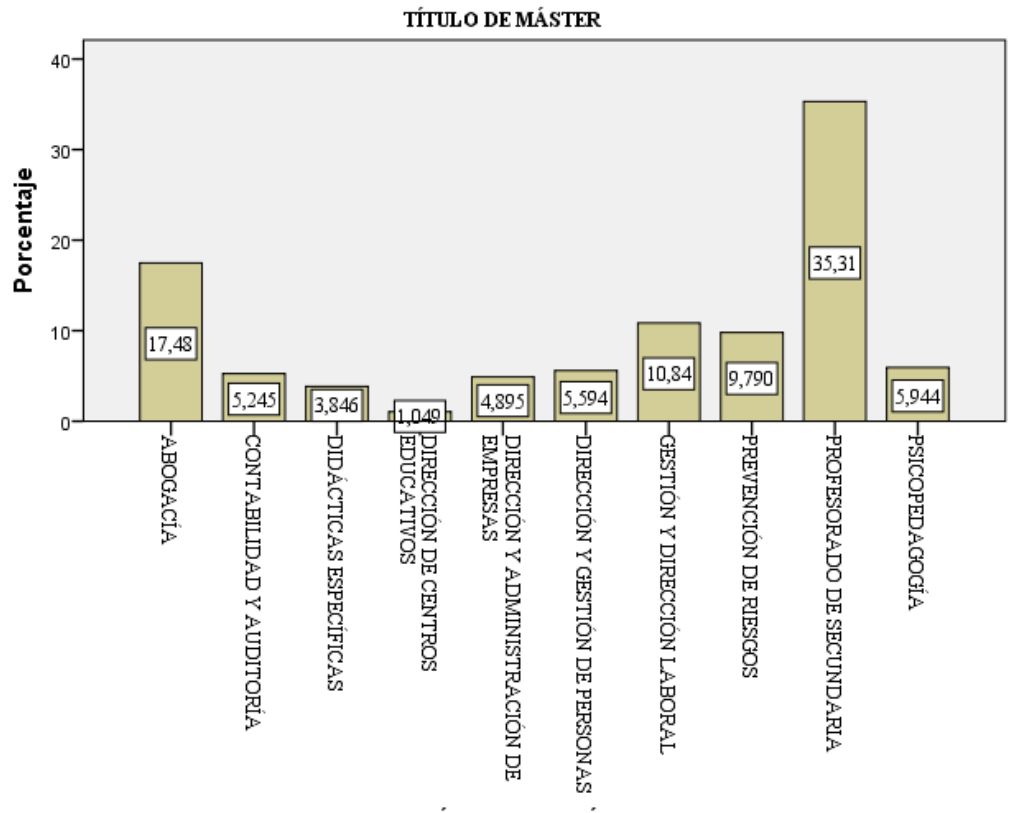

Figura 2. Títulos que cursa el alumnado de máster 
En relación al alumnado, es mayoritariamente femenino (64.7\%), con edades comprendidas entre los 17 y los 61 años, si bien la mayoría está en un intervalo entre 17 y 26 años, en el caso del grado y la mayoría en un intervalo entre 22 y 31 años en el caso de máster. Las principales fuentes utilizadas para elaborar los trabajos son los apuntes del profesorado (36.9\%) en el caso de grado y los libros y manuales (31.1\%) en el caso de máster y el soporte principal el electrónico (86.4\%). Un amplio número de alumnos/as indica tener formación sobre la citación de fuentes (61.9\%).

\section{INSTRUMENTO}

El Cuestionario de Atribuciones para la Detección de Coincidencias en Trabajos Académicos (CUDECO) se compone de preguntas referidas a variables identificativas, 39 ítems en escala Likert con siete alternativas de respuesta (totalmente en desacuerdo/totalmente de acuerdo), agrupados en cinco dimensiones: utilidad, carrera, causas, profesorado y compañeros/as; y una pregunta de carácter abierto, ¿Cómo crees que se puede evitar el plagio (copiar sin citar)? que puede relacionarse con cualquiera de las anteriores dimensiones. Los ítems a valorar en la dimensión Profesorado, son doce y se refieren a: instrucciones para elaborar el trabajo, normas para citar, seguimiento, atención individualizada en el aula y tutorías, evaluación, petición de trabajos teóricos/prácticos/creativos/innovadores, herramientas de detección de plagio, carga de trabajos y coordinación; todos ellos en relación con el marco teórico analizado.

Independientemente de haber revisado estudios anteriores sobre el tema, para analizar las prácticas de deshonestidad académica, se diseñó un cuestionario. En el actual proceso de validación (Muñoz-Cantero, Rebollo-Quintela, Mosteiro-García y Ocampo-Gómez, 2019), para la fiabilidad de las preguntas se ha empleado el coeficiente Alpha de Cronbach, que para el alumnado de la UDC presenta un valor de .868, siendo útil para lo que se pretende medir. Específicamente, la dimensión Profesorado para este alumnado presenta un índice de consistencia interna de .811. Sus aplicaciones previas y resultados parciales fueron descritos 
por Muñoz-Cantero y Espiñeira-Bellón (2019), Porto-Castro, Mosteiro-García y Gerpe-Pérez (2019a) y Porto-Castro et al. (2019b).

\section{RECOGIDA Y ANÁLISIS DE LA INFORMACIÓN}

El cuestionario fue administrado a la muestra del alumnado de la UDC para que lo contestase de manera anónima e individual. Los datos cuantitativos recopilados fueron sometidos a tratamiento estadístico empleando el paquete estadístico IBM SPSS Statistics en su versión 24.0. Asimismo, una vez recogidos los datos cualitativos y reducidos a través de distintas tareas de focalización, categorización, codificación y selección de la información obtenida, se procedió a la codificación de la información, lo que implica la asignación de un código a cada una de las categorías establecidas (Dorio, Sabariego, \& Massot, 2004; Tójar, 2006). En el proceso de análisis cualitativo, se empleó el programa MAXQDA, versión 2018.1.

\section{RESULTADOS}

En las tablas 1 y 2, se presenta el análisis descriptivo de la dimensión Profesorado, calculado a partir de la media de las puntuaciones obtenidas por el alumnado de grado y máster de la rama de Ciencias Sociales y Jurídicas:

Tabla 1. Estadísticos descriptivos de la dimensión profesorado en el alumnado de grado de la rama de Ciencias Sociales y Jurídicas

\begin{tabular}{ccccccccc}
\hline Dimensión & $\mathrm{N}$ & $\mathrm{M}$ & $\mathrm{Md}$ & $\mathrm{M} 0$ & $\mathrm{DT}$ & \multicolumn{3}{c}{ Percentiles } \\
\hline Profesorado & 1699 & 4.03 & 4 & 4 & 0.90 & 3.42 & 4.03 & 4.58 \\
\hline
\end{tabular}

El alumnado de grado tiende a situar las valoraciones acerca de las actuaciones del profesorado en categorías de respuesta intermedias $(M=4.03, M d=4.00, M 0=4.00)$ con cierta variabilidad de las respuestas $(\mathrm{DT}=0.90)$ respecto al valor medio. La concentración de las respuestas se produce entre 3 y $4\left(\mathrm{P}_{25}=3.42\right.$ y $\left.\mathrm{P}_{75}=4.58\right)$. 
Tabla 2. Estadísticos descriptivos de la dimensión profesorado en el alumnado de máster de la rama de Ciencias Sociales y Jurídicas

\begin{tabular}{ccccccccc}
\hline Dimensión & $\mathrm{N}$ & $\mathrm{M}$ & $\mathrm{Md}$ & $\mathrm{M} 0$ & $\mathrm{DT}$ & \multicolumn{3}{c}{ Percentiles } \\
\hline & & & & & & $\mathrm{P} 25$ & P50 & P75 \\
Profesorado & 286 & 3.92 & 4 & 4 & 1.06 & 3.17 & 4 & 4.58 \\
\hline
\end{tabular}

El alumnado de máster tiende a situar las valoraciones acerca de las actuaciones del profesorado en categorías de respuesta intermedias $(M=3.92, M d=4.00, M 0=4.00)$ con una mayor variabilidad de las respuestas que el alumnado de grado (DT=1.06) respecto al valor medio. La concentración de las respuestas se produce entre 3 y $4\left(\mathrm{P}_{25}=3.17\right.$ y $\left.\mathrm{P}_{75}=4.58\right)$.

En la tabla 3, se presenta el análisis descriptivo de la dimensión Profesorado, calculado a partir de la media de las puntuaciones obtenidas por parte del alumnado de grado y máster de los diferentes títulos de la rama de Ciencias Sociales y Jurídicas:

Tabla 3. Estadísticos descriptivos de la dimensión profesorado en el alumnado de grado y máster por títulos de la rama de Ciencias Sociales y Jurídicas

\begin{tabular}{|c|c|c|c|c|c|c|}
\hline Ittems & $\mathrm{N}$ (grado) & $\mathrm{M}$ & DT & $\mathrm{N}$ (máster) & $\mathrm{M}$ & DT \\
\hline Me da instrucciones precisas para elaborar el trabajo & 1699 & 4.57 & 1.30 & 286 & 4.29 & 1.56 \\
\hline $\begin{array}{l}\text { Me facilita las normas básicas para citar } \\
\text { adecuadamente }\end{array}$ & 1699 & 4.18 & 1.66 & 286 & 3.97 & 1.71 \\
\hline $\begin{array}{l}\text { Realiza un seguimiento de la evolución del trabajo } \\
\text { teniendo en cuenta la citación de fuentes }\end{array}$ & 1699 & 3.66 & 1.59 & 286 & 3.66 & 1.63 \\
\hline $\begin{array}{l}\text { Ofrece atención individualizada en el aula durante la } \\
\text { elaboración del trabajo }\end{array}$ & 1699 & 3.92 & 1.62 & 286 & 3.92 & 1.64 \\
\hline $\begin{array}{l}\text { Ofrece atención individualizada en las tutorías } \\
\text { durante la elaboración del trabajo }\end{array}$ & 1699 & 5.09 & 1.55 & 286 & 4.78 & 1.63 \\
\hline $\begin{array}{l}\text { Evalúa la correcta citación de las fuentes } \\
\text { documentales incorporadas }\end{array}$ & 1699 & 4.65 & 1.58 & 286 & 4.40 & 1.66 \\
\hline Pide trabajos esencialmente teóricos & 1699 & 4.66 & 1.43 & 286 & 4.52 & 1.60 \\
\hline Pide trabajos esencialmente prácticos & 1699 & 3.87 & 1.48 & 286 & 3.70 & 1.54 \\
\hline Pide trabajos creativos/innovadores & 1699 & 3.81 & 1.68 & 286 & 3.89 & 1.85 \\
\hline $\begin{array}{l}\text { Maneja herramientas de detección de fragmentos } \\
\text { copiados en los trabajos }\end{array}$ & 1699 & 4.00 & 1.70 & 286 & 3.86 & 1.84 \\
\hline $\begin{array}{l}\text { Adecúa la carga de trabajos al tiempo disponible } \\
\text { para cada asignatura }\end{array}$ & 1699 & 3.39 & 1.61 & 286 & 2.96 & 1.71 \\
\hline $\begin{array}{l}\text { Se coordina con los demás docentes para evitar } \\
\text { saturar de trabajos al alumnado }\end{array}$ & 1699 & 2.46 & 1.55 & 286 & 2.51 & 1.65 \\
\hline
\end{tabular}


Con respecto a las valoraciones del alumnado de grado sobre las acciones del profesorado encaminadas a evitar el plagio, destaca la alta valoración del ítem "Ofrece atención individualizada en las tutorías durante la elaboración del trabajo" $(M=5.09)$.

Con valores medios por encima de 4.5 estarían "Pide trabajos esencialmente teóricos" $(\mathrm{M}=4.66)$ "Evalúa la correcta citación de fuentes documentales incorporadas" $(M=4.65), y$ "Me da instrucciones precisas para elaborar el trabajo" $(M=4.57)$.

En orden descendente se hace referencia a los ítems "Me facilita las normas básicas para citar adecuadamente" ( $M=4.18)$, "Maneja herramientas de detección de fragmentos copiados en los trabajos" ( $(M=4.00)$, "Ofrece atención individualizada en el aula durante la elaboración del trabajo ( $M=3.92)$, "Pide trabajos esencialmente prácticos" (3.87), "Pide trabajos creativos/innovadores" ( $M=3.81)$ y "Realiza un seguimiento de la evolución del trabajo teniendo en cuenta la citación de fuentes" ( $M=3.66)$.

Por el contrario, hay que destacar la baja valoración del ítem "Se coordina con los demás docentes para evitar saturar de trabajos al alumnado" $(M=2.46)$ seguido por "Adecúa la carga de trabajo al tiempo disponible para cada asignatura" $(M=3.39)$, ambos por debajo de la media.

Con respecto a las valoraciones del alumnado de máster sobre las acciones del profesorado encaminadas a evitar el plagio, destaca la alta valoración del ítem "Ofrece atención individualizada en las tutorías durante la elaboración del trabajo" $(M=4.78)$.

Con valores medios por encima de 4, pero no tan próximos a 5 como el anterior, estarían "Pide trabajos esencialmente teóricos" ( $M=4.52)$, "Evalúa la correcta citación de fuentes documentales incorporadas" $(\mathrm{M}=4.40)$ y "Me da instrucciones precisas para elaborar el trabajo" ( $M=4.29)$.

En orden descendente se hace referencia a los ítems "Me facilita las normas básicas para citar adecuadamente" ( $M=3.97)$, "Ofrece atención individualizada en el aula durante la elaboración del trabajo $(M=3.92)$, "Pide trabajos creativos/innovadores" $(M=3.89)$, "Maneja herramientas de detección de fragmentos copiados en los trabajos" $(M=3.86)$, "Pide trabajos 
esencialmente prácticos" ( $M=3.70)$ y "Realiza un seguimiento de la evolución del trabajo teniendo en cuenta la citación de fuentes" ( $M=3.66)$.

Por el contrario, destaca la baja valoración del ítem "Se coordina con los demás docentes para evitar saturar de trabajos al alumnado" $(M=2.51)$ seguido por "Adecúa la carga de trabajo al tiempo disponible para cada asignatura" ( $\mathrm{M}=2.96)$, ambos por debajo de la media.

Con respecto al proceso cualitativo, en la categoría Profesorado se agrupan las respuestas centradas en las actuaciones del profesorado para evitar el plagio; este código agrupa las propuestas que versan sobre los diversos papeles que el profesorado puede y/o debe adoptar a la hora de evitar el plagio. Los subcódigos establecidos se recogen en la tabla 4.

Tabla 4. Categorías y subcategorías de análisis de la dimensión profesorado en el alumnado de la rama de Ciencias Sociales y Jurídicas de la UDC

\begin{tabular}{lll}
\hline Categoría & Subcategorías & \\
\hline Profesorado & Facilidades & $\begin{array}{l}\text { Propuestas en las que el profesorado facilita aspectos que podrían } \\
\text { ser útiles para evitar el plagio. } \\
\text { Propuestas en relación con las medidas de control, supervisión, } \\
\text { seguimiento, comprobación, revisión, tutorización,.... }\end{array}$ \\
Control & Explicación & $\begin{array}{l}\text { Propuestas relacionadas con la explicación de trabajos } \\
\text { académicos. }\end{array}$ \\
& Carga & $\begin{array}{l}\text { Propuestas ligadas a la carga, sobrecarga, saturación de trabajos, } \\
\text { horarios,... } \\
\text { Propuestas relacionadas con el reconocimiento o valoración } \\
\text { académica positiva, derivada del uso de la citación o no uso del } \\
\text { plagio. }\end{array}$ \\
\hline
\end{tabular}

\section{CONCLUSIONES}

El alumnado de grado y máster de la rama de Ciencias Sociales y Jurídicas de la UDC, pone de manifiesto, en primer lugar, que valora positivamente las actuaciones del profesorado para evitar el plagio ofreciendo atención individualizada en las tutorías de los trabajos de carácter normalmente teórico; ello indica el reconocimiento del alumnado por el trabajo académico del profesorado (Diez-Martínez, 2014). 
En segundo lugar, valora como aspectos positivos la correcta evaluación de las fuentes documentales que incorpora en sus trabajos, que el profesorado facilite instrucciones precisas para elaborar el trabajo o las normas básicas para citar adecuadamente. Otros/as autores/as como Sureda et al. (2009, p. 214) señalan igualmente la evitación de la "evaluación de los trabajos del alumnado de forma superficial y poco razonada" así como centrarse en evaluar todo el proceso y no únicamente el resultado (Universidad Rovira i Virgili, 2017). En cuanto a las instrucciones facilitadas, Sureda et al. (2009) indican que la poca claridad de éstas es otra de las causas atribuibles al plagio. Las cuestiones anteriores pueden relacionarse con las subcategorías Facilidades y Valoración propuestas por el alumnado que ha participado en el análisis de carácter cualitativo (aspectos útiles para evitar el plagio y propuestas para la valoración académica positiva).

En tercer lugar, con valores escasamente superiores a la media, el alumnado hace referencia positiva al tipo de trabajos que el profesorado solicita (prácticos/creativos/innovadores), a la atención prestada en el aula 0 a que el profesorado maneje herramientas de detección de fragmentos copiados en los trabajos (con ligeras variaciones entre grado y máster). El tipo de trabajos solicitados, el número y el diseño de las diferentes actividades es uno de los aspectos a tener en cuenta en la prevención del plagio (Sureda et al., 2009; Universidad Rovira i Virgili, 2017). En cuanto al seguimiento que se hace de éstos, Sureda et al. (2009) señalan que el plagio es atribuible además a la "liviana o escasa supervisión de los trabajos demandados" ( $p$. 212). Las cuestiones anteriores pueden relacionarse con la subcategoría Control propuesta por el alumnado que ha participado en el análisis de carácter cualitativo (en relación con las medidas de tutorización).

Finalmente, en cuarto lugar, el alumnado valora como aspectos negativos la no adecuación de la carga de trabajo al tiempo de cada asignatura y la falta de coordinación con los/las demás docentes con el fin de evitar la saturación de trabajos. Existen autores/as que hacen referencia a la sobrecarga de trabajos del alumnado como una de las causas más importantes de cometer plagio (Escalante, \& Ibarra, 2013; Lucía, Fernando, \& Castellanos, 2006; Sureda et 
al., 2009; Universidad Rovira i Virgili, 2017) seguida por "la descoordinación con los otros profesores del curso" (Sureda et al., 2009, p. 210). Las cuestiones anteriores pueden relacionarse con las subcategorías Explicación y Carga propuestas por el alumnado que ha participado en el análisis de carácter cualitativo (carga, sobrecarga, saturación de trabajos, horarios, ...).

Aunque existen un gran número de factores que deben ser tenidos en cuenta a la hora de planificar acciones orientadas a luchar contra el plagio, los resultados dan pie a prestar mayor atención al quehacer del profesorado a la hora de diseñar acciones, al igual que han ido manifestando los/las diferentes autores/as mencionadas/os, dado que la exigencia académica universitaria actual, fundamentada en "la falta de creatividad en el proceso de enseñanzaaprendizaje con formas de evaluación absurdas", muchas veces promueve la deshonestidad académica (Diez-Martínez, 2014, p. 15). La reformulación de los métodos de enseñanza parecen ir en la línea siguiente: potenciar una buena gestión del tiempo y de los recursos disponibles por parte del alumnado, solicitar los trabajos con suficiente antelación, poner más atención en la tipología de trabajos académicos solicitados explicando claramente además el sentido de la tarea solicitada, realizar un seguimiento de las tareas durante el proceso de desarrollo y diseñar actividades de evaluación que permitan realizar un seguimiento del proceso de aprendizaje del estudiantado, no exclusivamente el resultado final.

\section{REFERENCIAS}

Amador-Bautista, R. (2017). El plagio académico, transgresión de normas jurídicas, en publicaciones mexicanas de ciencias sociales y humanidades. Praxis Sociológica, 22, 155-167.

Bautista, F., Sánchez, P.A. \& Canto, P.J. (2017). Plagio en los postgrados de ciencias sociales en una universidad estatal de México. Revista Educación y Ciencia, 6(47), 82-97. 
Cebrián-Robles, V., Raposo-Rivas, M., Cebrián-de-la-Serna, M., \& Sarmiento-Campos, J.A. (2018). Percepción sobre el plagio académico de estudiantes universitarios españoles. Educación XXI, 21(2), 105-129.

Comas-Forgas, T. \& Sureda-Negre, J. (2016). Prevalencia y capacidad de reconocimiento del plagio académico entre el alumnado en el área de economía. El profesional de la información, 25(4), 616-622.

Diez-Martínez, E. (2015). Deshonestidad académica de alumnos y profesores. Su contribución en la desvinculación moral y corrupción social. Sinéctica, 44, 1-17.

Dorio, I., Sabariego, M. \& Massot, M.I. (2004). Características generales de la metodología cualitativa. En R. Bisquerra (Ed.), Metodología de la Investigación Educativa, (pp. 276292). Madrid: La Muralla.

Escalante, A.E. \& Ibarra, L.M. (2013). Dilemas que enfrenta el estudiantado de postgrado en su formación ética como investigador. Edetania, 43, 67-81.

García, M.P. \& García, M. (2012). Los métodos de investigación. En M.P. García \& P. Martínez (Coords.), Guía práctica para la realización de trabajos fin de grado y trabajos fin de máster (pp. 99-128). Murcia: Edit.um.

Hu, G. \& Sun, X. (2016). Conocimientos y actitudes ante el plagio del profesorado de lengua inglesa en universidades chinas. Comunicar, 48(24), 29-37.

Lucía, Fernando \& Castellanos (2006). Percepciones estudiantiles sobre el fraude académico: hallazgos y reflexiones pedagógicas. Revista de Estudios Sociales, 23, 37-44.

McMillan, J.H. \& Schumacher, S. (2005). Investigación educativa. Madrid: Pearson Educación.

Muñoz-Cantero, J.M. \& Espiñeira-Bellón, E.M. (2019). Percepción del alumnado sobre el uso de las citas en la realización de trabajos académicos. Trabajo presentado al XV Congreso Internacional Gallego-Portugués de Psicopedagogía, A Coruña.

Muñoz-Cantero, J.M., Rebollo-Quintela, N., Mosteiro-García, M.J., \& Ocampo Gómez, C.I. (2019). Validación del cuestionario de atribuciones para la detección de coincidencias en trabajos académicos. RELIEVE, 25(1), art. 4. 
Porto-Castro, A.M., Mosteiro-García, M.J. \& Gerpe-Pérez, E.M. (2019a). Las causas del plagio académico en estudiantes de Pedagogía. Actas del XIX Congreso Internacional de Investigación Educativa (vol. I).

Porto-Castro, A.M., Mosteiro-García, M.J. \& Gerpe-Pérez, E.M. (2019b). El plagio académico en la etapa universitaria: percepción del alumnado de Educación. Trabajo presentado al XV Congreso Internacional Gallego-Portugués de Psicopedagogía, A Coruña.

Rodríguez, M.J. (2007). Espacio Europeo de Educación Superior y metodologías docentes activas. Alicante: Universidad de Alicante.

Sanvicén, P., \& Molina, F. (2015). Efectos del uso de Internet como fuente principal de información. Evidencias en estudiantes de primer curso universitario. Revista de Investigación Social, 15, 352-386.

Sureda, J., Comas, R. \& Morey, M. (2009). Las causas del plagio académico entre el alumnado universitario según el profesorado. Revista Iberoamericana de Educación, 50, 197-220.

Sutherland-Smith, W. (2010). Retribution, deterrence and reform: the dilemmas of plagiarism management in universities. Journal of Higher Education Policy and Management, 32 (1), 5-16.

Tójar, J.C. (2006). La investigación cualitativa: comprender y actuar. Madrid: La Muralla.

Universidad Rovira i Virgili (2017). Prevención, detección y tratamiento del plagio en la docencia. Guía para el profesorado. Tarragona: Universidad Rovira i Virgili.

Williams, K. \& Carroll, J. (2009). Referencing \& Understanding Plagiarism. Basingstoke: Palgrave Macmillan.

\footnotetext{
' El presente trabajo se ha realizado en el marco de un contrato con la Xunta de Galicia financiado por la Secretaría General de Universidades "Estudio sobre el plagio en estudiantes del Sistema Universitario de Galicia", con referencia 002/2019.
} 